
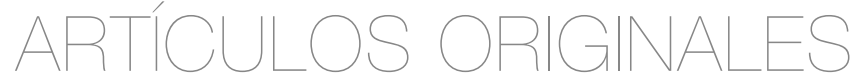

\title{
La capacitación en pequeñas y medianas empresas: hacia una caracterización
}

\author{
DANIELA LÓPEZ MONTALVO* \\ EYLIN JORGE COTO** \\ AYDÉ CADENA LÓPEZ***
}

* Maestra en Ingeniería Industrial. Unidad Profesional Interdisciplinaria de Ingeniería y Ciencias Sociales y Administrativas, Instituto Politécnico Nacional, Ciudad de México, México. E-mail: dlopezm1815@alumno.ipn.mx. ORCID: 0000-0002-4403-4793.Google Scholar: https://scholar.google.com/citations?hl=es\&user=3qgvMmoAAAJ.

** Maestra en Ingeniería Industrial. Unidad Profesional Interdisciplinaria de Ingeniería y Ciencias Sociales y Administrativas, Instituto Politécnico Nacional, Ciudad de México, México. E-mail: ejorgec180o@alumno.ipn.mx. ORCID: 000o-0001-9109-1339. Google Scholar: https://scholar.google.com/citations?hl=es\&user=_7gCpHAAAAAJ.

*** Doctora en Estudios Organizacionales. Unidad Profesional Interdisciplinaria de Ingeniería y Ciencias Sociales y Administrativas, Instituto Politécnico Nacional, Ciudad de México, México. E-mail: aydecadena@gmail.com. ORCID: 0000-0001-6387-5873.Google Scholar: https://scholar.google.com/citations?hl=es\&user=nK62J-8AAAAJ. 
COMO CITAR ESTE ARTÍCULO

How to cite this article:

López, D., Jorge, E. y Cadena, A. (2021). La capacitación en pequeñas y medianas empresas: hacia una caracterización. Revista Perspectiva Empresarial, 8(1), 71-85.

Recibido: 09 de diciembre de 2020 Aceptado: 02 de marzo de 2021
RESUMEN Las pequeñas y medianas empresas son protagonistas del desarrollo económico de los países por sus aportes en ingresos y generación de empleos. No obstante, enfrentan diversos inconvenientes que les impiden desarrollarse plenamente. Objetivo. Realizar un análisis de literatura sobre la capacitación en pequeñas y medianas empresas. Metodología. Se realizó una revisión sistemática de artículos para elaborar una codificación por categorías, procesando la información con el apoyo de matrices. Resultados. La revisión arrojó elementos que caracterizan a la capacitación en pequeñas y medianas empresas: poca visión estratégica; exiguo apoyo gubernamental y renuencia de dueños/gerentes para implementarla. Conclusión. Esta revisión ha demostrado que es menester explorar o generar estrategias para una capacitación efectiva y una innovación de los modelos actuales de capacitación para estas empresas.

PALABRAS CLAVE capacitación, pequeña empresa, estudio bibliográfico, gestión del personal, administración.

\section{Training in small and medium-sized enterprises: towards a characterization}

ABSTRACT Small and medium-sized enterprises are key players in the economic development of countries due to their contributions in terms of income and employment generation. However, they face several drawbacks that prevent them from developing fully. Objective. To conduct a literature review on training in small and medium-sized enterprises. Methodology. A systematic review of articles was carried out to create a codification by categories, processing the information with the support of matrices. Results. The review revealed elements that characterize training in small and medium-sized enterprises: little strategic vision; limited governmental support and owner's/managers' reluctance to implement it. Conclusions. This review has shown there is a need to explore or generate strategies for effective training and innovation of current training models for these companies.

KEYWORDS Training, small business, literature review, personnel management, administration. 


\section{Formação em pequenas e médias empresas: para uma caracterização}

RESUMO As pequenas e médias empresas são protagonistas do desenvolvimento econômico dos países por suas contribuições para a geração de renda e empregos. No entanto, eles enfrentam várias desvantagens que os impedem de se desenvolver totalmente. Objetivo. Realizar uma análise da literatura sobre formação em pequenas e médias empresas. Metodologia. Foi realizada uma revisão sistemática dos artigos para elaboração de uma codificação por categorias, processando as informações com o apoio de matrizes. Resultados. A revisão rendeu elementos que caracterizam a formação em pequenas e médias empresas: pouca visão estratégica; escasso apoio governamental e relutância do proprietário/gerente em implementá-lo. Conclusões. Esta revisão mostrou que é necessário explorar ou gerar estratégias de formação e inovação eficazes dos atuais modelos de formação para essas empresas.

PALAVRAS CHAVE treinamento, pequena empresa, estudo bibliográfico, gestão de pessoas, administração. 


\section{Introducción}

Las pequeñasy medianas empresas - PyMEstienen gran importancia en la economía. En la Unión Europea conforman el 99 \% de las empresas existentes, cubren dos tercios del mercado laboral y aportan el $60 \%$ del valor agregado (Arasti, Zandi and Talebi, 2012). Y contribuyen al crecimiento económico local, nacional e internacional con la generación de ingresos, empleos y productos (Dang et al., 2018; Rabie, Cant and Wiid, 2016); no obstante, su tasa de fracaso es alta (Rabie, Cant and Wiid, 2016). Ncube y Zondo (2018) afirman que alrededor del $75 \%$ de las nuevas PyMEs no llegan a convertirse en negocios formales, incluso en países desarrollados (como, por ejemplo, Reino Unido, Estados Unidos o Francia) solo el 30 \% logran sobrevivir en sus primeros cinco años de vida (Bai, Yuan and Pan, 2017). De ahí que la probabilidad de que sobrevivan en sus etapas iniciales es menor a las empresas globales (Ncube and Zondo, 2018), dejándolas vulnerables en los mercados competitivos (Bai, Yuan and Pan, 2017).

A pesar de su trascendencia económica las características, implicaciones y razones de éxito o fracaso de las PyMEs han sido poco estudiadas en países en desarrollo como México (De la Rosa, Rivera y Marín, 2015). En este sentido, por ejemplo, la indagación sobre la importancia de la gestión de recursos humanos - GRH - para estas empresas ha sido exigua; no obstante, "la literatura existente detecta que las prácticas de gestión de recursos humanos tienen efectos significativos en la productividad, el rendimiento y la innovación" (Omar, Arokiasamy and Ismail, 2009, p. 222).

La GRH es necesaria para las PyMEs, ya que permite el desarrollo de mejores habilidades y mayores conocimientos (Omar, Arokiasamy and Ismail, 2009). Sin embargo las prácticas de GRH en estas empresas se caracterizan por su escasa implementación, formalidad y evaluación tal como es el caso de la capacitación (Walker et al., 2007). Para algunos autores, la capacitación no suele estar disponible en las PyMEs a diferencia de las empresas grandes (Rabie, Cant and Wiid, 2016; Susomrith and Coetzer, 2015) en las que suele haber recursos disponibles para ella (Damoah, Ashie and Kodjo-Kekesi, 2016).
Con base en lo anterior, el objetivo de este artículo es realizar una revisión de literatura para identificar y caracterizar la capacitación en las PyMEs. El texto se divide en cinco apartados: el primero establece un marco de referencia en el que se definen características de las PyMEs y se presentan argumentos sobre la importancia de las prácticas de GRH, específicamente de la capacitación. El segundo delimita la metodología para la búsqueda, selección y revisión de la literatura. El tercero presenta los resultados obtenidos, divididos por categorías de análisis. El cuarto contiene la discusión de los resultados, definiendo cómo es la capacitación en las PyMEs. A manera de conclusión, se discuten limitaciones y alcances del trabajo.

\section{Marco de referencia}

Las PyMEs son entidades económicas resultantes de la asociación de cierto número de personas que buscan cumplir objetivos comunes a través de actividades laborales en coordinación (Biondic, 2020). Estas empresas son pilares elementales en el desarrollo económico de los países -en especial, en vías de desarrolloporque permiten crear nuevos empleos a nivel local, mejorar la distribución del ingreso y contribuir al crecimiento de las exportaciones. Aquello impacta positivamente en la reducción de la pobreza y en el desarrollo de la industria, economías rurales y emprendimientos sociales (Ramukumba, 2014).

Una característica principal de las PyMEs es el número de integrantes, generalmente son menos de cincuenta (Ghayoomi et al., 2020). Aunado a sus moderados volúmenes de producción en comparación con empresas grandes (De la Rosa, Rivera y Marín, 2015), ya que suelen enfrentarse a la limitación de recursos y al uso de tecnologías simples (Agwu and Afieroho, 2016). Sus procesos productivos son sencillos y se basan en gestiones flexibles y creativas, lo que les permite acoplarse a tales limitaciones. En parte, aquello se debe a que prevalece una lógica familiar y a que la gestión y la propiedad se concentran en una persona o en un grupo pequeño (De la Rosa, Rivera y Marín, 2015).

Además se caracterizan por una relación informal entre empleadores y empleados (Agwu 
and Afieroho, 2016). Los dueños o dirigentes tienen fuerte control de la información y los procesos, centralizando la toma de decisiones (De la Rosa, Rivera y Marín, 2015). Otras especificidades son: poca delimitación de funciones; cambio constante en las actividades de producción; falta de reglas o políticas internas y rotación constante del personal (Marcelino-Aranda, Robles y Baldazo, 2015). Si bien aquella flexibilidad es importante para su adaptación al cambio, también puede ser un obstáculo interno para su crecimiento y consolidación (Pacheco, 2015).

En este sentido diversos estudios han revelado que varias problemáticas que enfrentan las PyMEs se relacionan con los recursos humanos - RRHHtal como la incapacidad de retener al personal y no contar con mano de obra especializada o calificada (Sethi, 2018). Shafeek (2016) argumenta que la meta de la GRH en las PyMEs es alcanzar la eficiencia en el manejo del capital humano, lo que implica formar trabajadores calificados y competentes. De ahí que la GRH sea un reto para estas empresas, pues gran parte de su éxito depende de esto.

Por ello la formación de RRHH es elemental para lograr ventajas competitivas sostenibles en las PyMEs (Pérez-Bustamante et al., 2016) dado que permiten incrementar el rendimiento de los gerentes, seleccionar al personal conveniente, mejorar las capacidades y habilidades de los empleados, mejorar la productividad y generar o transferir nuevas tecnologías (Shafeek, 2016). Para Steijvers, Lybaert y Dekker (2017) cuando las prácticas de RRHH como el reclutamiento, la evaluación y la compensación son planeadas e implementadas formalmente, contribuyen al mejoramiento del desempeño general de las PyMEs. A lo que Dang et al. (2018) suman el trabajo en equipo, el pago de incentivos a los trabajadores y la capacitación. Por tal motivo la GRH ha cobrado relevancia en la sobrevivencia de estas organizaciones (Pérez-Bustamante et al., 2016) porque contribuye a su productividad, rendimiento e innovación (Dang et al., 2018).

No obstante, existe escasa literatura sobre la importancia de la GRH para las PyMEs en países en desarrollo (Dang et al., 2018). Los pocos estudios existentes afirman que en estas organizaciones la GRH es informal y que los empleados aprenden sobre la marcha (Tam and Gray, 2016). Antonioli y
Della Torre (2015) aseguran que esas circunstancias y la relevancia de las PyMEs en las economías, brindan argumentos para profundizar en la GRH en estas empresas y así poder generarse estrategias de capacitación diseñadas especialmente para estas. Ante esto, el presente estudio realiza una revisión de literatura para mostrar cómo es la capacitación en las PyMEs. A continuación, se muestra la metodología utilizada.

\section{Metodología}

Se revisaron artículos científicos en tres etapas: en la primera se realizó una búsqueda de publicaciones que abordan el tema dela capacitación en PyMEs. Las bases de datos consideradas fueron Web of Science, Scopus y EBSCO; su alto rigor científico valida la calidad del conocimiento que ofrecen sus artículos. En dichos índices, primero se buscaron los términos small and medium businesses training y small and medium enterprises training.

Debido a que solo se encontraron 16 artículos en dicha búsqueda, se realizó una segunda con los términos training + SMEs; hallando otros 49 artículos. Ninguna búsqueda fue limitada a un período específico, por lo que todas las publicaciones encontradas hasta el 30 de marzo de 2020 fueron consideradas. Así pues, se obtuvo un total de 65 artículos.

La segunda etapa fue de selección. Allí se revisaron los títulos, resúmenes y palabras clave de los 65 artículos encontrados para verificar la pertinencia de las investigaciones respecto al objetivo de esta. Se seleccionaron 50 artículos en función de su pertinencia y relevancia para el tema aquí tratado y se descartaron 15 trabajos cuyo contenido no respondía al interés del estudio.

Seleccionados los 50 artículos, siguió la etapa de revisión y codificación. Primero las autoras leyeron y revisaron cinco artículos cada una; para, a partir de una codificación abierta, establecer un primer conjunto de categorías de análisis. Posteriormente se realizó una comparación y se discutió sobre el primer grupo de categorías. Derivado de la discusión se definió un segundo grupo de categorías de análisis a partir del cual se realizó la revisión de 
los 50 artículos. Cabe mencionar que durante la revisión surgieron otras categorías no consideradas previamente, pero que dada su importancia se incorporaron al resultado final.

La fase de revisión y codificación de los artículos se hizo a través de una matriz en la que se vació la información obtenida por categoría por medio de un análisis de contenidos. Antes de iniciar con la redacción del texto, las autoras revisaron y discutieron detalladamente los resultados obtenidos. A partir de esto, algunas categorías cambiaron de nombre o se fusionaron debido a la naturaleza o semejanza de la información. Los resultados obtenidos se presentan enseguida.

\section{Resultados}

En este apartado se presentan las principales consideraciones encontradas en la revisión de los 50 artículos científicos. Dichos resultados son ordenados por categoría analítica, allí se exponen los argumentos examinados en cada publicación.

Para mostrar los resultados es conveniente definir qué es capacitación, ya que en la revisión se detectaron diversas definiciones. La capacitación o formación - como también reconoce la literaturaes definida como un proceso enmarcado en la GRH. Por ello se relaciona con actividades como la planificación de la carrera, la evaluación del desempeño, el reclutamiento y la selección (PérezBustamante et al., 2016).

La capacitación se divide en tres vertientes principales: el aprendizaje teórico conocimientos-; el aprendizaje práctico habilidades - y el cambio de actitud por parte de los involucrados en estas actividades (Antonioli and Della Torre, 2016; Vásquez, 2018). Así, se enfoca en desarrollar competencias específicas (Coetzer, Wallo and Kock, 2019) en los colaboradores de una organización con el fin de disminuir la brecha entre el desempeño del trabajador y los requerimientos del puesto de trabajo (Vásquez, 2018).

La formación también es un conjunto de actividades sistemáticas y planificadas que toman lugar dentro y fuera de la empresa, destinadas a trabajadores de toda la organización (Bager et al., 2015; Susomrith, Coetzer and Ampofo, 2019). Vásquez (2018) agrega la necesidad de conformar procesos de capacitación que alineen los intereses empresariales con los personales, buscando una situación de ganar-ganar para los participantes. En una parte prolifera la motivación de los trabajadores por capacitarse (Ncube and Zondo, 2018) y para la empresa se consideran beneficios tales como mayor rentabilidad, mayor productividad, mejora de los procesos internos entre otros. Ante esto, Pauli (2020) y Marenichenko (2016) sostienen que la capacitación en las PyMEs debe estar sustentada en el conocimiento científico para lograr resultados de calidad.

\section{Beneficios para las PyMEs}

Un gran beneficio de la formación en las PyMEs es el desarrollo de competencias laborales en todos los niveles organizacionales (Bai, Yuan and Pan, 2017; Nai-Wen, Chih-Yun and Lin, 2008). A mayor calidad del capital humano, mayor flexibilidad y capacidad de respuesta ante las variaciones internas de la organización y los cambios que impone el entorno (Karim, 2019; Kotey and Folker, 2007). No obstante, el propósito de la formación en las altas esferas en las PyMEs deviene de la necesidad de adquirir conocimientos de gestión organizacional y desarrollar habilidades para una toma de decisiones certera (Ghayoomi et al., 2020; Rafiki, 2020).

De igual manera Kessy y Temu (2010) mencionan que parte de la formación está enfocada en el cambio de actitudes de los gerentes con referencia a cómo perciben y dirigen sus negocios. Aquello permite que los directores de las PyMEs cambien el espectro visual de una administración estrecha hacia un pensamiento estratégico. Por ello Antonioli y Della Torre (2015) destacan la capacitación como un elemento decisivo en la adopción del enfoque estratégico, lo que deriva en una ventaja competitiva (Coetzer, Wallo and Kock, 2019; Omar, Arokiasamy and Ismail, 2009). Esto mejora los procesos internos (Dang et al., 2018; Pauli, 2020), aumenta el rendimiento empresarial (Nai-Wen, Chih-Yun and Lin, 2008; Saastamoinen, Reijonen and Tammi, 2017), incrementa la productividad (Dang et al., 2018; Pauli, 2020), la rentabilidad (Rabie, Cant and Wiid, 2016) y la calidad de sus productos, servicios y procesos (Agwu 
and Afieroho, 2016; Kholopane, 2016). En este sentido la capacitación también ayuda a enfrentar los cambios organizacionales y tecnológicos tanto intencionados como los impuestos por el mercado (Omar, Arokiasamy and Ismail, 2009; Sethi, 2018).

La capacitación formal es vista por los colaboradores como un factor que incide en su motivación laboral (Bai, Yuan and Pan, 2017; Omar, Arokiasamy and Ismail, 2009), aumentando el compromiso con la empresa (Pauli, 2020; Susomrith, Coetzer and Ampofo, 2019) y disminuyendo la rotación laboral (Rabie, Cant and Wiid, 2016). Las organizaciones que crean ambientes de trabajo enfocados a la mejora continua y con una cultura organizacional de apoyo tienen mayor probabilidad de que los colaboradores sugieran y promuevan proyectos de innovación (Ilkhamova et al., 2017; Pauli, 2020; Sethi, 2018). Aquellos factores son determinantes en la supervivencia (Kotey and Folker, 2007) y sostenibilidad (Arasti, Zandi and Talebi, 2012; Kholopane, 2016) de las PyMEs. Kotey y Slade (2005) mencionan la importancia de las inducciones tanto a la empresa como al puesto de trabajo. Este tipo de formación permite esclarecer las pretensiones de la organización para con el trabajador y las facilidades que puede ofrecerles.

\section{Visión estratégica}

La visión estratégica refiere a la planeación y alcance de objetivos a largo plazo. En las PyMEs esto no es común por su naturaleza, su orientación informal o flexible y su preocupación por la supervivencia o rendimiento a corto plazo (Kotey and Folker, 2007; Saastamoinen, Reijonen and Tammi, 2017). Debido a que la capacitación suele presentar resultados visibles y tangibles en el largo plazo esta no es prioridad en las PyMEs (Shafeek, 2016), pues prefieren enfocarse en cumplir con la nómina, aunque esto no las deslinda de la responsabilidad de desarrollar talento humano (Ngaochay and Walsh, 2017).

Lo anterior, se relaciona con que muchas PyMEs "están dirigidas por dueños/gerentes que carecen de visión a largo plazo, [...] necesaria paraidentificar la dirección futura de su negocio" (Mazzarol, 2004, p. 1). Esto destaca la necesidad de la capacitación en todos los niveles organizacionales, ya que es importante que cambie la visión de las PyMEs con respecto a la capacitación. Resulta necesario que esta se incluya en su planeación estratégica y se considere para enriquecer las habilidades, aumentar el conocimiento de su fuerza laboral y mejorar el rendimiento de los colaboradores y de la organización en general (Khan and Ramsey, 2013).

\section{Estructura del proceso de capacitación}

Para Pauli (2020) las PyMEs deben profesionalizar y formalizar la capacitación a través de modelos alineados a investigaciones científicas. Los autores aconsejan cuatro etapas para ello: detección de necesidades de capacitación -DNC-, planificación, ejecución y evaluación. Por su parte Vásquez (2018) recomienda estructurar la capacitación basado en el ciclo administrativo: planificación, organización, ejecución y evaluación. Esta propuesta coincide con la de Pauli (2020) puesto que la fase de organización de Vásquez (2018) incluye su etapa de DNC.

Respecto a la primera etapa, Kotey y Folker (2007) afirman que escasamente las PyMEs realizan análisis formales sobre las necesidades de capacitación. Sin embargo Nai-Wen, Chih-Yun y Lin (2008) recalcan la importancia de este análisis dada la relación implícita con los resultados obtenidos tras la implementación. Debido a la informalidad arraigada en estas empresas, las evaluaciones del desempeño pueden utilizarse para identificar las falencias a ser resueltas con la capacitación (Roy and Raymond, 2008).

En la segunda fase ambos autores mencionan la planificación de los recursos materiales y económicos, los participantes, el objetivo y los procedimientos para llevar a cabo las acciones de formación. En consecuencia, Simpson, Schraeder y Borowski (2015) exponen que el resultado de esta etapa consiste en un plan de formación que debe contemplar: objetivo, participantes, método de capacitación, materiales, encargado, presupuesto, disponibilidad de tiempo y lugar destinado para el desarrollo de la formación.

Por su parte Sirinaga, Khatibi y Azam (2020) sugieren comprobar la efectividad de la capacitación con el modelo de Kirkpatrick por su alta utilización en la literatura. Esa propuesta establece cuatro niveles de análisis: reacción de los trabajadores; aprendizaje adquirido; cambio del comportamiento de las personas involucradas 
y resultados económicos. Aunque un aspecto negativo prevaleciente en los procesos de formación es la poca seriedad en las actividades de evaluación y seguimiento de la formación, lo cual dificulta definir con claridad los beneficios de la inversión (Ngaochay and Walsh, 2017).

\section{Formalidad en los procesos de capacitación}

La capacitación informal existe en las PyMEs, independientemente de su tamaño (Kotey and Folker, 2007), y es utilizada en mayor o menor medida según sea necesario (Roy and Raymond, 2008). Aun cuando vasta literatura justifica los beneficios de la formalidad (Shafeek, 2016) el comportamiento de las PyMEs está sesgado por la informalidad, la falta de sistematicidad y la poca planificación en procesos de capacitación (Coetzer, Wallo and Kock, 2019; Padachi and Lukea-Bhiwajee, 2016; Sethi, 2018).

Esto se refleja en la baja asistencia de los trabajadores a eventos formales de capacitación (Susomrith, Coetzer and Ampofo, 2019), prefiriendo la flexibilidad de la capacitación informal (Adla, Gallego-Roquelaure and Calamel, 2019; Sethi, 2018). Jayawarna, Macpherson y Wilson (2007) justifican esto basándose en tres ideas: se incurre en un menor gasto; se ajusta a la baja disponibilidad de tiempo de los trabajadores o a las tareas diarias y se enfoca en las necesidades específicas de los trabajadores. Con base en ello Tam y Gray (2016) exponen que la informalidad en los procesos de formación de las PyMEs le da cierta flexibilidad a los dueños, quienes escogen este método de capacitación por considerarlo más apegado a su realidad (Adla, Gallego-Roquelaure and Calamel, 2019; Bager et al., 2015).

\section{Método de capacitación}

Los métodos de capacitación son formales o informales y las PyMEs prefieren uno u otro, dependiendo del nivel de formalidad en su gestión (Kotey and Folker, 2007). Los formales incluyen la impartición de cursos, talleres, instrucciones guiadas, seminarios, tutorías, e-learning y conferencias (Bager et al., 2015; Nagi, 2008; Simpson, Schraeder and Borowski, 2015; Sirinaga, Khatibi and Azam, 2020). Esta capacitación puede realizarse al interior o en espacios ajenos a la empresa (Antonioli and Della Torre, 2015).

En la capacitación informal los ejemplos más citados son: capacitación durante el trabajo; rotación de labores; aprendizaje basado en experiencia; desarrollo de habilidades con prácticas laborales e interacción con compañeros más experimentados (Kotey and Folker, 2007; Sulistyowati et al., 2018; Susomrith, Coetzer and Ampofo, 2019). También el tamaño de la organización puede ser determinante para escoger el tipo de capacitación. Existe mayor probabilidad que las empresas pequeñas opten por métodos menos formales, en contraste con las empresas medianas que se apoyan más en métodos formales (Kotey and Slade, 2005; Susomrith, Coetzer and Ampofo, 2019).

Hoy el e-learning constituye un método de capacitación formal (Roy and Raymond, 2008), reconocido por la flexibilidad del horario y el lugar para las acciones de formación (Nagi, 2008). Asimismo, se destaca por las comodidades que ofrece tanto al aprendiz como a la organización. No obstante, un elemento que puede atentar en su contra es la falta de tecnologías informáticas y de conectividad (Nagi, 2008).

\section{Pertinencia del programa de capacitación}

La formación debe ser una oportunidad igualitaria para todos los niveles de la organización. En este sentido Rabie, Canty Wiid (2016) mencionan la importancia del acceso a los programas de capacitación que permitan desarrollar diferentes tipos de competencias. Sin embargo Lange, Ottens y Taylor (2000) destacan limitantes de la capacitación como el acceso y la provisión de los programas, afirmando que el diseño del contenido - en ciertas ocasiones- no está alineado a las necesidades de las PyMEs (Kotey and Folker, 2007; Nai-Wen, ChihYun and Lin, 2008).

Relacionado a esto, está la baja disponibilidad de formación proveniente de fuentes externas (Bai, Yuan and Pan, 2017) y la inexistencia de programas de capacitación diseñados especialmente para las PyMEs (Padachi and Lukea-Bhiwajee, 2016). Ante esta situación los gerentes/dueños optan por crearlos (Tam and Gray, 2016), dependiendo de las necesidades contemporáneas sin contemplar 
el rigor científico necesario para establecer un programa de formación apegado a estándares de calidad (Pauli, 2020). Así, la informalidad y la carencia de enfoque a la mejora continua repercuten en la pertinencia de los programas de capacitación (Khan and Ramsey, 2013).

\section{¿Gasto o inversión?}

Comúnmente las PyMEs no destinan un presupuesto periódico para capacitación (Ferraris et al., 2000) dado que es un tema poco valorado por sus dueños/gerentes. Algunos consideran que es un gasto innecesario (Ngaochay and Walsh, 2017) y que su implementación no tendrá beneficios económicos (Panagiotakopoulos, 2011; Rabie, Cant and Wiid, 2016). De igual manera está el temor de invertir en la formación de los empleados y que una vez que estos adquieran nuevas capacidades y habilidades decidan abandonar la empresa.

Por su parte Kessy y Temu (2010) sugieren que la capacitación en PyMEs es una inversión que aumenta las posibilidades de mejorar la productividad y los ingresos empresariales, así como cumplir los objetivos estratégicos (Nai-Wen, Chih-Yun and Lin, 2008). Igualmente se trata de una herramienta que podría posicionar a estas empresas sobre sus competidores (Vásquez, 2018) y permitir "sobrepasar la incertidumbre en la toma de decisiones" (Kessy and Temu, 2010, p. 110).

\section{Disponibilidad de presupuesto y fuentes de financiamiento externo}

Para Kotey y Folker (2007) pocas PyMEs destinan una parte de su presupuesto para capacitación. Aun cuando contemplan dicha partida, la misma está marcada por la escasez de recursos financieros; lo anterior, imposibilita que su capital humano se desarrolle continua y formalmente (Ferraris et al., 2000; Saastamoinen, Reijonen and Tammi, 2017). En este sentido Shafeek (2016) afirma que hay fuentes de financiamiento externo como medio para las PyMEs, pero hay desinformación sobre cómo adquirir tales subvenciones (Ramukumba, 2014). Shafeek (2016) comenta que, si se recibe financiamiento externo, es importante aprovechar al máximo el monto donado; para ello se propone definir un proceso de capacitación formal que garantice el retorno proveniente de dicha inversión (Agwu and Afieroho, 2016; Simpson, Schraeder and Borowski, 2015).

\section{Apoyo gubernamental}

Las PyMEs son fundamentales en la economía de los países (Muller et al., 2015; Rabie, Cant and Wiid, 2016), pero tienen acceso limitado a financiación de entidades gubernamentales (Kessy and Temu, 2010). Sin embargo algunos gobiernos imponen procesos de capacitación formal a estas empresas, volviéndose una presión financiera y legal más que una motivación (Padachi and Lukea-Bhiwajee, 2016). Teniendo las PyMEs tanta relevancia para la economía a nivel mundial, resulta necesario prestar mayor atención económica a sus estrategias de innovación y capacitación (Antonioli and Della Torre, 2015). Para esto, Panagiotakopoulos (2011) propone un esquema de garantía de préstamos por parte del gobierno con el fin de financiar el desarrollo de recursos humanos. También apunta que "este esquema puede ser realmente útil para empresas que han sido rechazadas para préstamos en otros lugares" (p. 16).

Abor y Quartey (2010) recomiendan a los gobiernos de los países incitar a instituciones de capacitación y ciertas entidades no gubernamentales por medio de incentivos fiscales a brindar capacitación a empresarios de las PyMEs. De ahí que es indispensable que los gobiernos aboguen por mayor educación y capacitación para estas empresas, por lo que sus políticas deberían enfocarse en reducir las limitaciones y mejorar la provisión de dicha capacitación (Bai, Yuan and Pan, 2017).

\section{Búsqueda de asesoría externa para la capacitación}

Algunas fuentes externas son las empresas privadas de capacitación, grupos industriales, asociaciones comerciales, organismos profesionales, universidades y organizaciones respaldadas por el gobierno (Bai, Yuan and Pan, 2017; Bager etal., 2015). Pauli (2020) aconseja acudir a las asesorías externas cuando las PyMEs no estén en disposición deasumir la capacitación o cuando detecten una necesidad que requiera un grado de especialización superior al desarrollado por la empresa (Pérez-Bustamante et al., 2016). Sin embargo Panagiotakopoulos (2011) menciona la desconfianza de los dueños de estas empresas ante las capacitaciones externas, 
lo que deriva en la poca utilización de este recurso (Sulistyowati et al., 2018).

Por otro lado, Antonioli y Della Torre (2015) sostienen que las organizaciones a cargo de ofrecer asesoría y capacitación a las PyMEs deben determinar con precisión las necesidades de estas empresas y saber distinguirlas de las organizaciones grandes; más aún, cuando el presupuesto de las primeras es limitado.

\section{Problemas técnicos}

Es común que las PyMEs utilicen tecnologías simples que no requieren conocimientos avanzados (Sulistyowati et al., 2018), razón por la cual consideran de menor importancia la capacitación. Adicional a las restricciones en los procesos de RRHH (Ghayoomi et al., 2020) se tiene la falta de financiamiento de tecnología (Ramukumba, 2014) y como consecuencia el poco acceso a cursos a distancia (Nagi, 2008). Esto, aunado a la escasez de habilidades de gestión (Arasti, Zandi and Talebi, 2012), impide el desarrollo de una capacitación exitosa.

Otro problema al implementar procesos de capacitación es su complejidad. La mayoría de los programas de formación son elaborados y dirigidos a grandes empresas (Susomrith and Coetzer, 2015). Sulistyowati et al. (2018) destacan la necesidad de que tales programas sean adaptados a las características y problemas particulares de las PyMEs y así permitirles un mayor desarrollo.

\section{Percepción de los dueños/gerentes de PyMEs}

Los dueños/gerentes de PyMEs tienen disímiles concepciones erróneas respecto a la capacitación. Frecuentemente su actitud es negativa y renuente ante ella (Sulistyowati et al., 2018; Susomrith and Coetzer, 2015), además de considerarla un lujo innecesario e inasequible (Kotey and Slade, 2005). Asimismo, es habitual la idea de que la capacitación es solo viable para empresas grandes (Damoah, Ashie and Kodjo-Kekesi, 2016). A esto se suma la poca disponibilidad de tiempo y la dificultad para relevar a los colaboradores mientras están capacitándose (Saastamoinen, Reijonen and Tammi, 2017).
Resulta fundamental analizar aquellas percepciones puesto que son las que determinan si la capacitación es propicia o no, la manera en la que debería realizarse y quiénes tendrán acceso a ella. Una circunstancia que influye en la decisión del dueño/gerente para ofrecer formación es el deseo de resultados a corto plazo (Pachadi and LukeaBhiwajee, 2016), loqueimpide que una capacitación formal sea la opción preferida. La falta de evidencia sobre las ventajas a corto plazo dificulta definir su utilidad para la empresa (Susomrith and Coetzer, 2015). Para Walker et al. (2007) es importante "establecer suficiente evidencia de la relación entre la participación en capacitación, el incremento en las habilidades gerenciales y el mejoramiento del desempeño del negocio" (p. 295) para una mayor confianza de los dueños/gerentes.

También es necesario brindar alternativas para acceder a procesos de capacitación, aportando vías para mejorar su desarrollo. Una opción es la creación de centros virtuales de aprendizaje donde se impartan seminarios especializados en las necesidades de las PyMEs (Panagiotakopoulos, 2011). Roy y Raymond (2008) sugieren desarrollar una cultura de estudio virtual o e-learning que permita la capacitación de los empleados y de los dueños/gerentes de las PyMEs sin necesidad de salir de la empresa e incurrir en gastos adicionales.

\section{Habilidades gerenciales}

Los gerentes tienen bastante injerencia en la toma de decisiones respecto a la capacitación en su empresa (Damoah, Ashie and Kodjo-Kekesi, 2016). No obstante, la falta de habilidades en planeación estratégica puede impedir que se elija el camino correcto. Como destaca Johnson (2002) es común que estas personas no cuenten con la destreza ni experiencia para identificar las necesidades de capacitación del personal, además son renuentes a la asesoría externa.

Para Jayawarna, Macpherson y Wilson (2007) la falta de habilidades de gestión en las PyMEs es un tema clave y una prioridad por resolver. La capacitación no solo es necesaria para el personal general, sino también para los gerentes dado su papel trascendental en el desarrollo de estas empresas. Con base en ello Arasti, Zandi y Talebi (2012) afirman que la capacitación gerencial contribuye al refuerzo de habilidades como la 
gestión de crisis, el marketing financieroy la gestión del talento humano, lo que ayuda a mejorar la toma de decisiones. Por medio de la capacitación formal los gerentes también pueden ampliar sus redes de negocio, transferir tecnología y desarrollar entidades comerciales. Asimismo, la capacitación puede traer crecimiento en las utilidades dado un mejor desempeño del gerente en la implementación deideas para el aumento de la productividad (Kessy and Temu, 2010).

Por su parte un beneficio adicional de la capacitación es la creación de un entorno organizacional que permita la difusión y regeneración continua del conocimiento y la innovación, induciendo al aprendizaje constante y acumulativo (Antonioli and Della Torre, 2015). Del mismo modo Susomrith, Coetzer y Ampofo (2019) sostienen que la capacitación está positivamente relacionada con la satisfacción laboral y el compromiso afectivo de los colaboradores con la empresa.

\section{Disposición de los trabajadores}

Los trabajadores son la población objetivo de la capacitación, lo cual no significa que siempre estén dispuestos a recibirla. Panagiotakopoulos (2011) argumenta que la ausencia de un vínculo claro y demostrable entre la capacitación y factores como el aumento de sus ingresos o de su desarrollo profesional genera poca motivación de los trabajadores para la formación. Además las altas cargas de trabajo y la falta de tiempo representan barreras para los procesos en cuestión (Susomrith and Coetzer, 2015). No obstante, la poca disposición no es general; Royy Raymond (2008) sostienen que muchos empleados, por elección propia, deciden capacitarse a través del e-learning.

Otro panorama común es que los colaboradores desean recibir capacitación efectiva y suficiente en la pyme que laboran, pero la empresa no cuenta con una estructura organizacional adecuada o políticas que la promuevan (Tam and Gray, 2016). Dada esta situación, muchos empleados deciden aprender de manera autónoma o a través de los proyectos en los que se desempeñan (Susomrith and Coetzer, 2015).

Lo anterior, no anula la existencia de PyMEs que realmente se esfuerzan por capacitar a sus empleados. Bai, Yuan y Pan (2017) encontraron que diferentes directores ejecutivos de este tipo de empresas en China consideraban la capacitación como un incentivo importante para sus empleados. Observaron que aquellos que tenían mayor participación en procesos de capacitación se encontraban más comprometidos con la empresa y motivados en su trabajo. Asimismo, presentaban menor probabilidad de dejarla o tener comportamientos perjudiciales. Al respecto, Simpson, Schraeder y Borowski (2015) refieren que para que un proceso de capacitación tenga mayor probabilidad de éxito es fundamental considerar las opiniones y percepciones de los trabajadores.

\section{Mercados internacionales}

Las empresas -incluidas las PyMEs- buscan adaptarse a la globalización para continuar en el mercado, por lo que deben enfrentar diferentes presiones competitivas tal como la participación en proyectos de capacitación que les permitan convertirse en empresas de clase mundial (Roy and Raymond, 2008). Independientemente del esfuerzo que requiere ello representa un beneficio para las PyMEs dados los nuevos conocimientos, capacidades y habilidades que deben adquirir para competir en un mercado global (Nai-Wen, Chih-Yun and Lin, 2008; Sethi, 2018).

Las instituciones internacionales también han hecho un llamado a las PyMEs y al mercado para aumentar las habilidades de su personal, en especial a través dela capacitación formal (Antonioli and Della Torre, 2015). Acatar propuestas como esta, hace que dichas empresas mejoren su competitividad y sea mayor la probabilidad de éxito en el contexto de la globalización. Además de desarrollar o mejorar habilidades y capacidades técnicas, la capacitación también debería estar enfocada en herramientas innovadoras para responder a las necesidades del mercado (Vásquez, 2018).

\section{Discusión}

Los artículos analizados arrojaron elementos que caracterizan a la capacitación en las PyMEs como una práctica que aún no es considerada imperante en estas empresas por razones diversas: (i) desconocimiento de beneficios; (ii) 
desconocimiento de entidades proveedoras de formación; (iii) programas disponibles (Damoah, Ashie and Kodjo-Kekesi, 2016); (iv) renuencia de los propietarios a invertir en la formación ante el temor de que los trabajadores decidan marcharse; (v) baja disponibilidad de tiempo (Susomrith and Coetzer, 2015) y (vi) capacitación poco constante e informal que imposibilita ver los resultados esperados en el corto plazo (Saastamoinen, Reijonen and Tammi, 2017).

El bajo rendimiento de los trabajadores es otro factor por el cual las PyMEs prefieren no invertir en capacitación. Ante la limitación de recursos, las empresas no pueden permitirse capacitar a empleados con bajo rendimiento; de ahí que prefieren conformar normativas legales y administrativas para el despido; desde la perspectiva de los dueños/gerentes de las PyMEs, esto conlleva menos inversión que la capacitación (Kotey and Slade, 2005). Así pues, estas empresas carecen de incentivos suficientes para considerar la capacitación como un proceso elemental para su competitividad; prefieren contratar personal que ya cuente con los conocimientos y capacidades necesarias (Roshchin and Travkin, 2017).

En este sentido puede concluirse que la capacitación, en especial la formal, no es una práctica común en las PyMEs. Su ausencia representa un gran obstáculo interno en estas empresas, impidiendo su crecimiento y contribuyendo a sus altas tasas de fracaso (Saastamoinen, Reijonen and Tammi, 2017). Implementar procesos de capacitación con base en las propuestas mencionadas ayuda a obtener mayor profesionalización (Pauli, 2020) y calidad. Asimismo, la ausencia o poca implementación de capacitación formal genera otros inconvenientes como la alta rotación de personal y bajas perspectivas de crecimiento a largo plazo.

Así pues, las PyMEs enfrentan diferentes desafíos y barreras cuando se refiere a la capacitación. En esta investigación se ha visto que cuando se trata de estas empresas, la capacitación se encuentra en estado de infancia (Padachi and Lukea-Bhiwajee, 2016). Esto se refleja en la escasez de estudios sobre el tema a pesar de la relevancia de las PyMEs para las economías mundiales y de los RRHH como fuente de sobrevivencia. Lo anterior, otorga argumentos suficientes para aumentar la investigación sobre la capacitación en las PyMEs.
Esta revisión ha demostrado que es menester explorarogenerar estrategias para una capacitación efectiva y una innovación de los modelos actuales de capacitación para estas empresas.

\section{Conclusión: limitaciones y alcances de la investigación}

Esta investigación ha representado un primer esfuerzo de las autoras por caracterizar una imagen al día de hoy, de la capacitación en las PyMEs. Al ser un primer ejercicio, el estudio presentalimitaciones; la principal es el tamaño de la muestra de la literatura analizada. Por tal motivo, esta revisión abre una futura agenda en la que es menester realizar una segunda investigación de este corte que considere otro tipo de literatura (como, por ejemplo, capítulos de libro, obras completas y tesis) que verse sobre este tema.

El trabajo también tiene ciertos alcances que resultan pertinentes. El primero es que permite obtener un panorama que, a partir de ciertos elementos, muestra la situación de la capacitación en las PyMEs. Este aporte resulta considerable ante los pocos estudios realizados sobre el tema, pues en la búsqueda realizada en tres bases de datos distintas solo se encontraron 65 trabajos de los cuales únicamente 50 abordan directamente la temática en cuestión.

Si bien el tema de la capacitación ha sido ampliamente estudiado con respecto a las grandes empresas, los artículos encontrados en esta búsqueda demuestran que no es así en el caso de las PyMEs. Así que esta investigación aporta al estudio y a la integración de un panorama sobre la capacitación en estas empresas. Por ello este estudio puede ser útil no solo en términos del debate académico, sino también para propietarios y gerentes de PyMEs puesto que se identifican y sintetizan diversas problemáticas que pueden atenderse para lograr procesos de capacitación exitosos. 


\section{Referencias}

Abor, J. and Quartey, P. (2010). Issues in SME development in Ghana and South Africa. International Research Journal of Finance and Economics, 39, 218-228.

Adla, L., Gallego-Roquelaure, V. and Calamel, L. (2019). Human resource management and innovation in SMEs. Personnel Review, 49(8), 1519-1535.

Agwu, M.E. and Afieroho, E. (2016). Impact of product quality management on the growth of small and medium sized enterprises. European Journal of Social Sciences, 52(2), 175-190.

Antonioli, D. and Della Torre, E. (2015). Innovation adoption and training activities in SMEs. International Journal of Human Resource Management, 27(3), 311-337.

Arasti, Z., Zandi, F. and Talebi, K. (2012). Exploring the effect of individual factors on business failure in Iranian new established small businesses. International Business Research, 5(4), 2-11.

Bager, T.E. etal.(2015). Enrollment of SME managers to growth-oriented training programs. International Journal of Entrepreneurial Behavior \& Research, 21(4), 578-599.

Bai, Y., Yuan, J.J. and Pan, J. (2017). Why SMEs in emerging economies are reluctant to provide employee training: Evidence from China. International Small Business Journal: Researching Entrepreneurship, 35(6), 751-766.

Biondic, D. (2020). Integralni Indeks Učinkovitosti Malog I Srednjeg Drvno Industrijskog Proizvodnog Poduzeća Finalnih Proizvoda. Šumarski List, 144(1-2), 75-81.

Coetzer, A., Wallo, A. and Kock, H. (2019). The owner-manager's role as a facilitator of informal learning in small businesses. Human Resource Development International, 22(5), 420-452.
Damoah, O.B., Ashie, A. and Kodjo-Kekesi, E. (2016). The propensity to participate in formal training programmes: Evidence from small and medium-sized enterprises (SMEs) in Ghana. World Journal of Entrepreneurship, Management and Sustainable Development, 12(4), 344-358.

Dang, T. et al. (2018). Human resource management practices and firm outcomes: evidence from Vietnam. Journal of Asian Business and Economic Studies, 25(2), 221-238.

Dela Rosa, A., Rivera, I. y Marín, A. (2015). Propuesta de clasificación integral de la Mipyme basada en la complementariedad disciplinar de las perspectivas económica, administrativa y organizacional. En Rivera, I. y De la Rosa, A. $L a$ Mipyme a debate. Perspectivas de estudio, mitos, actores clave, propuestasy vinculación (pp.3-49). Ciudad de México, México: Competitive Press.

Ferraris, M. et al. (2000). Managing the change from face to-face to distance training for SMEs. Computers \& Education, 34(2), 77-91.

Ghayoomi, M. et al. (2020). Designing a model for creation of export consortia in business cluster. International Journal of Engineering, 33(3), 459-467.

Ilkhamova, M.U. et al. (2017). The problems of professional training of practice-oriented specialists for small enterprises of footwear and leather production industries in Uzbekistan. IOP Conference Series: Materials Science and Engineering, 254(23), 1-4.

Jayawarna, D., Macpherson, A. and Wilson, A. (2007). Training commitmentand performance in manufacturing SMEs: Incidence, intensity and approaches. Journal of Small Business and Enterprise Development, 14(2), 321-338.

Johnson, S. (2002). Lifelong learning and SMEs: issues for research and policy.Journal of Small Business and Enterprise Development, 9(3), 285-295.

Karim, R. (2019). Impact of different training and development programs on employee performance in Bangladesh perspective. 
International Journal of Entrepreneurial Research, 1(2), 8-14.

Kessy, S. and Temu, S. (2010). The impact of training on performance of micro and small enterprises served by microfinance institutions in Tanzania. Research Journal of Business Management, 4(2), 103-111.

Khan, S. and Ramsey, P. (2013). Easy, economic, expedient-An effective training evaluation model for SMEs. In Green, A. (Ed.), 10th International Conference on Intellectual Capital, Knowledge managementICICKM (pp.540-551), Washington, USA: The George Washington University.

Kholopane, P. (2016). The impact of total quality management system on sustainability of small and medium businesses in South Africa. IAMOT, 1934-1942.

Kotey, B. and Folker, C. (2007). Employee training in SMEs: Effect of size and firm type-Family and nonfamily. Journal of Small Business Management, 45(2), 214-238.

Kotey, B. and Slade, P. (2005). Formal human resource management practices in small growing firms. Journal of Small Business Management, 43(1), 16-40.

Lange, T., Ottens, M. and Taylor, A. (2000). SMEs and barriers to skills development: a Scottish perspective. Journal of European Industrial Training, 24(1), 5-11.

Marcelino-Aranda, M., Robles, C. y Baldazo, F.A. (2015). El apoyo a las micro y pequeñas empresas, entre la propuesta oficial y la aptitud del empresario. Estudio de caso. En Rivera, I. y De la Rosa, A. La Mipyme a debate. Perspectivas de estudio, mitos, actores clave, propuestas y vinculación (pp. 195-208). Ciudad de México, México: Competitive Press.

Marenichenko, V. (2016). Mechanisms of state regulation of small and medium businesses. Baltic Journal of Economic Studies, 2(5), 41-47.

Mazzarol, T. (2004). Strategic management of small firms: a proposed framework for entrepreneurial ventures. In 17th Annual SEAANZ Conference, Queensland University of Technology, Brisbane, Australia.

Muller, P. et al. (2015). Annual Report on European SMEs 2014/2015. Brussels, Belgium: European Commission.

Nagi, K. (2008). Use of moodle reports for knowledge management, planning and eTraining in SMEs. In 4th IEEE International Conference on Management of Innovation and Technology, Bangkok, Thailand.

Nai-Wen, C., Chih-Yun, W. and Lin, C.Y. (2008). Does training facilitate SME's performance? The International Journal of Human Resource Management, 19(10), 1962-1975.

Ncube, T.R. and Zondo, R.W. (2018). Influence of self-motivation and intrinsic motivational factors for small and medium business growth: A South African case study. SAJEMS. South African Journal of Economic and Management Sciences, 21(1), 1-7.

Ngaochay, T. and Walsh, J. (2017). Upgrading employees' skills in Thai SMEs sustainably. Pacific Business Review, 9(9), 129-136.

Omar, S.S., Arokiasamy, L. and Ismail, M. (2009). The background and challenges faced by the small and medium enterprises. A human resource development perspective. International Journal of Business and Management, 4(10), 95-102.

Pacheco, A.A. (2015). La dirección integral de Mypime: mitos, premisas y propuestas. En Rivera, I. y De la Rosa, A. La Mipyme a debate. Perspectivas de estudio, mitos, actores clave, propuestasy vinculación (pp. 241-259). Ciudad de México, México: Competitive Press.

Padachi, K. and Lukea-Bhiwajee, S. (2016). Barriers to employee training in small and medium sized enterprises: Insights and evidences from Mauritius. European Journal of Training and Development, 40(4), 232-247.

Panagiotakopoulos, A. (2011). Barriers to employee training and learning in small and medium- 
sized enterprises (SMEs). Development and Learning in Organizations, 25(3), 15-18.

Pauli, U. (2020). Training professionalisation and SME performance. Human Resource Development International, 23(2), 168-187.

Pérez-Bustamante, G.O. et al. (2016). The impact of continuous training in small and medium enterprises: Lessons from an industrial case analysis. Journal of Business Economics and Management, 17(2), 234-250.

Rabie, C., Cant, M.C. and Wiid, J.A. (2016). Training and development in SMEs: South Africa's key to survival and success? Journal of Applied Business Research, 32(4), 1009-1024.

Rafiki, A. (2020). Determinants of SME growth: an empirical study in Saudi Arabia. International Journal of Organizational Analysis, 28(1), 205225.

Ramukumba, T.(2014). Overcoming SME challenges through critical success factors: A case of SMEs in the Western Cape Province, South Africa. Economic and Business Review, 16(1), 19-38.

Roshchin, S. and Travkin, P. (2017). Determinants of on-the-job training in enterprises: the Russian case. European Journal of Training and Development, 41(9), 758-775.

Roy, A. and Raymond, L. (2008). Meeting the training needs of SMEs: Is e-learning a solution? Electronic Journal of e-Learning, 6(2), 89-98.

Saastamoinen, J., Reijonen, H. and Tammi, T. (2017). The role of training in dismatling barriers to SME participation in public procurement. Journal of Public Procurement, 17(1), 1-30.

Sethi, A. (2018). Obstacles faced by small medium enterprises in imparting training: An empirical study on textile industry. Pacific Business Review International, 10(10), 103-109.

Shafeek, H. (2016). The impact of human resources management practices on SMEs performance in Romania. ANNALS of the Faculty of Engineering Hunedoara-International Journal of Engineering, 14(4), 91-102.
Simpson, J., Schraeder, M. and Borowski, M. (2015). Enhancing the value of training: creating closer time linkages between training acquisition, application and compensation. Development and Learning in Organizations, 29(5), 16-18.

Sirinaga, I.P., Khatibi, A. and Azam, S.F. (2020). The effect of knowledge managementand employee training on performance if small and mediumscaled enterprises (SMEs): Employee training as a mediator. European Journal of Human Resource Management Studies, 4(1), 1-21.

Steijvers, T., Lybaert, N. and Dekker, J. (2017). Formal human resource practices in family firms. Journal of Family Business Management, $7(2), 151-165$.

Sulistyowati, L. et al. (2018). Development of small and medium business (SMES) of mango dodol processing to increase the added value (a case study in Ujungjaya Village, Indramayu District, West Java). IOP Conference Series: Earth and Environmental Science, 142, 1-8.

Susomrith, P. and Coetzer, A. (2015). Employees' perceptions of barriers to participation in training and development in small engineering businesses. Journal of Workplace Learning, 27(7), 561-578.

Susomrith, P., Coetzer, A. and Ampofo, E. (2019). Training and development in small professional services firms. European Journal of Training and Development, 43(5/6), 517-535.

Tam, S. and Gray, D.E. (2016). The practice of employee learning in SME workplaces: A micro view from the life-cycle perspective. Journal of Small Business and Enterprise Development, 23(3), 671-690.

Vásquez, M. (2018). Factorial analysis of the elements that compose the training in the small and medium enterprises of the Industrial sector. Management, 22(2), 42-59.

Walker, E. et al. (2007). Small business owners: too busy to train? Journal of Small Business and Enterprise Development, 14(2), 294-306. 